\title{
On the Influence of the Pauli Principle on Atomic Nuclei
}

\author{
H. H. Hackenbroich, H. G. Wahsweiler, and K. Wildermuth \\ Institut für Theoretische Physik der Universität Tübingen \\ (Z. Naturforschg. 21 a, 870-880 [1966]; received 21 March 1966)
}

Professor Dr. W. Gentner zum 60. Geburtstag gewidmet

\begin{abstract}
In this paper we discuss the influence of the PAULI principle on some properties of nuclear systems consisting of a cluster surrounded by many other nucleons; to do this we use two models. We find that, though transition probabilities may be influenced drastically, the general features of the energetical behaviour of such systems is practically unaffected by the exclusion principle. The consequences for real nuclei are discussed: For instance, closed shells remain energetically favoured even if surrounded by many other nucleons.
\end{abstract}

It has often been discussed ${ }^{1}$ that both heavy and light nuclei show properties which may be explained most naturally as a consequence of internal clustering of the nucleons. Examples are the existence of $\alpha$-clusters in the nuclear surface and the occurrence of asymmetric fission.

Whereas for light nuclei many detailed calculations exist which show this clustering explicitly, we do not have similar calculations for heavy nuclei because of computational difficulties. Therefore we treat here simplified models to show firstly how the PAULI principle may generally influence some nuclear properties very drastically (density, transition amplitudes) and secondly that the energetical behaviour of clusters surrounded by many other nucleons is essentially unchanged. The investigation of this second point is quite important for the understanding of asymmetric fission.

We shall pursue these questions with the aid of two models: In section II we will analyse a two $\alpha$-cluster state; in section III we consider a onedimensional cluster embedded in nuclear matter; in section IV we will apply our results.

\section{The $\alpha-\alpha$-state}

We want to examine a model nucleus being a bound state of two $\alpha$-clusters. We describe an $\alpha$ particle by a wave function built according to the oscillator shell model

$$
\begin{aligned}
& \varphi_{a}(1,2,3,4)=\frac{64}{\sqrt{4 !}}\left(\frac{2 a}{\pi}\right)^{9 / 2} \\
& \alpha(1) \bar{\alpha}(1) \ldots \beta(1) \bar{\beta}(1)
\end{aligned}
$$

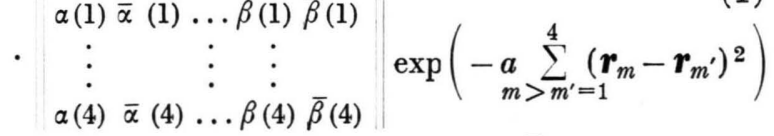

$$
\begin{aligned}
& \text { ( } \alpha, \beta \text { denoting spin states, } \bar{\alpha} \text { and } \bar{\beta} \text { isospin states). }
\end{aligned}
$$

If we would neglect antisymmetrization between the $\alpha$-clusters, we could describe the $\alpha$ - $\alpha$ bound state by the function

$$
\begin{array}{r}
\Phi(1, \ldots, 8)=\varphi_{a}(1,2,3,4) \varphi_{a}(5,6,7,8) \frac{(16 b)^{n+3 / 2}}{\Gamma(n+3 / 2)} \\
\cdot R^{n} e^{-8 b R^{2}} Y_{L, M}\left(\Omega_{R}\right)
\end{array}
$$

$\boldsymbol{R}$ being the difference coordinate of the two $\alpha$ clusters:

$$
R=\frac{1}{4}\left(\sum_{m=1}^{4} \boldsymbol{r}_{m}-\sum_{m=5}^{8} \boldsymbol{r}_{m}\right) .
$$

The function $\Phi$ of eq. (2) is an eigenfunction to spin and isospin with eigenvalue zero and to the angular momentum. But the function (2) violates the PaUli principle which the wave function of the composite nucleus has to fulfill. So we have to construct from $\Phi$ another function $\Psi$ :

$$
\begin{aligned}
\Psi & =N \mathfrak{U}^{\prime}\{\Phi\} \equiv N\left(\Phi+\sum_{P^{\prime}}(-1)^{P^{\prime}} P^{\prime}\{\Phi\}\right), \\
N & \equiv N(a, b, L, M, n)
\end{aligned}
$$

where $P^{\prime}$ means all permutations which permute nucleons between different clusters; $P^{\prime}\{\Phi\}$ is the function $\Phi$ with permutated coordinates; $N$ means a normalization constant. $\Psi$ is antisymmetric in all particle coordinates. In the case $b=a$ the wave function $\Psi$ is an ordinary oscillator shell model function; for $b \ll a$ the two $\alpha$-clusters are far apart and we expect

$$
\left\langle P^{\prime}\{\Phi\} \mid \Phi\right\rangle \ll 1
$$

for all ${ }^{2} P^{\prime}$; so in this case the antisymmetrization

1 Conf. K. Wildermuth, W. McClure, To be published in Springer Tracts, Ergeb. exakt. Naturw.

2 This is not quite true in this special case. For example, the permutation of all nucleons of cluster 1 against all nucleons of cluster 2 will give the original function (for even $L)$. So the overlap 0 defined by (6) goes to $1 / 4$ for $b / a \rightarrow 0$ and not to zero. 
between the clusters will be quite unimportant, contrary to the case $b=a$. In order to discuss the effect of antisymmetrization further we need a quantitative measure for the degree of overlapping of the clusters; this is given by the quantity

$$
0=\left|\frac{\langle(\Psi-\Phi) \mid \Phi\rangle}{\langle\Psi \mid \Phi\rangle}\right|^{2} .
$$

$0 \ll 1$ means that the antisymmetrization is unimportant.

Let us now make some general arguments as to how the degree of overlapping depends upon the mutual spatial penetration of the clusters. Suppose first that the two clusters do not penetrate each other, that is, the most probable separation distance of the cluster centroids is much larger than the sum of the "radii" of the two clusters. In this case $P^{\prime}\{\Phi\}$ as a function of the permuted nucleon coordinates will be small in a region where $\Phi$ as a function of the nucleon coordinates is large, and vice versa. Consequently $O$ will be much less than unity. Suppose on the other hand the most probable separation distance of the clusters is extremely small compared to the sum of the radii of the clusters. By the uncertainty principle the kinetic energy of the two clusters about one another then becomes very large. In this semi-classical limit situation we again expect the influence of the PAULI principle between the clusters to be small. To see that this is in fact so we consider the cluster function in the momentum representation. We see that the internal momentum spread within a cluster is much smaller than the momentum spread of the relative motion ${ }^{4}$. Hence $P^{\prime}\{\Phi\}$ as a function of the permuted nucleon momenta is small where $\Phi$ as a function of the nucleon momenta is large, and vice versa. Hence the degree of overlapping, $O$ will again be small despite the considerable spatial penetration ${ }^{5}$. Between these two extremes $O$ will become large and we expect the behaviour of the antisymmetrized function to deviate considerably from the ordinary particle picture.

Our arguments are not limited to the special form of the wave functions (2) and (4); but we shall verify our arguments for these wave functions

3 This second extreme will not be realized in nature because in this case the internal cluster structure would be destroyed.

4 This is obvious, for the width parameters in the momentum representation are proportional to the reciprocals of the respective width parameters in the position representation. quantitatively. To do this, we calculate from the wave functions (2) and (4) the charge density defined by ${ }^{6}$

$\varrho^{\mathrm{D}}(r)$

$=4 \sum_{s_{m}} \sum_{t_{m}} \int \mathrm{d} \Omega_{\boldsymbol{r}} \mathrm{d} \boldsymbol{r}_{2} \ldots \mathrm{d} \boldsymbol{r}_{8} \delta\left(\frac{1}{8} \sum_{m=1}^{8} \boldsymbol{r}_{m}\right) \Phi^{*} \Phi$

or by

$\varrho^{A}(r)$

$=4 \sum_{s_{m}} \sum_{t_{m}} \int \mathrm{d} \Omega_{\boldsymbol{r}} \mathrm{d} \boldsymbol{r}_{2} \ldots \mathrm{d} \boldsymbol{r}_{8} \delta\left(\frac{1}{8} \sum_{m=1}^{8} \boldsymbol{r}_{m}\right) \Psi^{*} \Psi$

respectively. We call $\varrho^{\mathrm{D}}(r)$ the "direct" charge density, whereas $\varrho^{\mathbf{A}}(r)$ is the charge density calculated with the help of the correctly antisymmetrized wave function. In (7) and (8) we use

$$
\begin{aligned}
\boldsymbol{r} & =\boldsymbol{r}_{1}-\frac{1}{8} \sum_{m=1}^{8} \boldsymbol{r}_{m}, \quad n=4, \quad L=M=0, \\
a & =0.05 \cdot 10^{-26} \mathrm{~cm}^{-2} .
\end{aligned}
$$

The sums should run over all spin and isospin coordinates. The results of these calculations are shown in Fig. $1-3$, with $b / a$ as parameter. Inspection of formulas (1) and (2) will show that $b / a$, which is the ratio of the relative motion and internal $\alpha$-cluster width parameters, is a rough measure of the mutual spatial penetration of the two $\alpha$-clusters.

For small mutual presentation of the clusters, $b / a=1 / 4$, the antisymmetrization practically does not influence the charge distribution. For large penetration, $b / a=1$ and $b / a=4$, the charge distribution is altered considerably. The change is alway in the direction of reducing the charge density for small $r$-values and increasing it at larger $r$-values. This is understandable for when the clusters begin to overlap the PAULI principle forces nucleons of the same spin and isospin to repel each other. For $b / a$ much greater than 5 in our example the charge distributions again become essentially the same for both the unsymmetrized and antisymmetrized wave function. The behaviour of the charge density is thus just what we had expected from our general arguments.

5 Cf. Footnote ${ }^{3}$.

6 The $\delta$-functions in the integrals (7) and (8) are necessary because the functions (2) and (8) depend on 7 "internal" coordinate vectors only. 

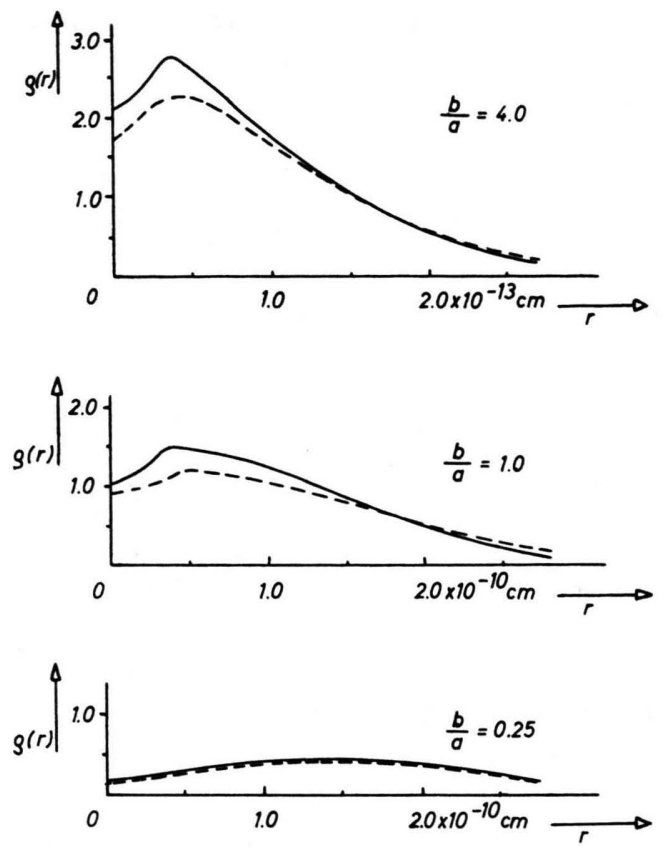

Figs. $1-3$. Dotted line: charge distribution $\varrho^{\mathrm{A}}(r)$ of the completely antisymmetrized wave function (4) calculated from eq. (8). Solid line: charge distribution $\varrho^{\mathrm{D}}(r)$ of the unsymmetrized wave function (8), calculated from eq. (7).

Next we compare the elastic quadrupole transition probabilities $|Q(\mathrm{E} 2)|^{2}$ from the $2^{+}$level to the ground state as a function of the cluster penetration $b / a$, again for the unsymmetrized wave function (2) and the antisymmetrized wave function (4). We have for

$$
\begin{aligned}
& |Q(\mathrm{E} 2)|^{2} \\
& \quad=\frac{15}{32} \frac{e^{2}}{\pi}\left|\left\langle I_{\text {fin }}\left|\sum_{m=1}^{8}\left(x_{m}+i y_{m}\right)^{2} \frac{1+\tau_{z_{m}}}{2}\right| I_{\text {in }}\right\rangle\right|^{2}
\end{aligned}
$$

where $\left|I_{\text {fin }}\right\rangle$ and $\left|I_{\text {in }}\right\rangle$ denote the final (ground) state and the initial $\left(2^{+}\right)$state, respectively.

In Fig. 4 we plot the quantity

$$
Q=\left|\frac{Q(\mathrm{E} 2)}{Q^{0}(\mathrm{E} 2)}\right|^{2}
$$

as a function of $b / a ;\left|Q^{0}(\mathrm{E} 2)\right|^{2}$ is the transition probability for the oscillator model value $b / a=1$. For the wave functions of the $2^{+}$excited state and $\mathrm{O}^{+}$ground state we use the functions of eq. (2) and (4) with just $L$ changed between the two states; again we choose $n=4$.

We see that the transition probability calculated from the unsymmetrized wave function (2) is a rather smooth function of $b / a$ and does not approach

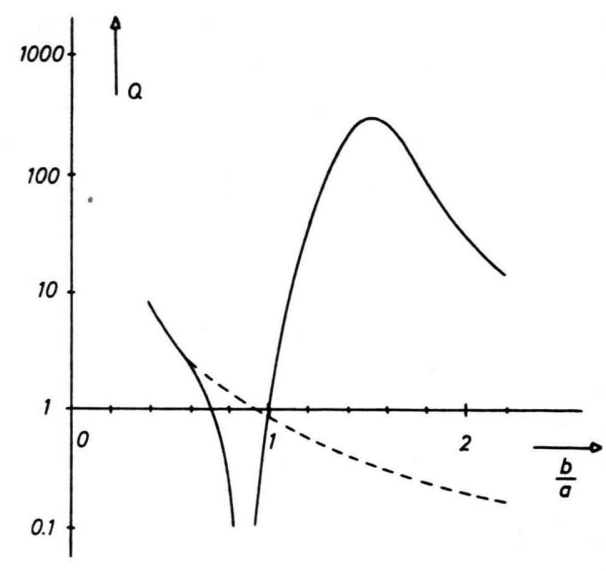

Fig. 4. Behaviour of the quadrupole transition probability $Q$ defined by eq. ( 9 a), with increasing cluster separation $b / a$. Solid line: $Q$ calculated with the antisymmetrized wave function (4) ; Dotted line: $Q$ calculated with the unsymmetrized wave function (2).

zero for any finite value of $b / a$. On the other hand, the transition probability computed from the correctly antisymmetrized function (4) goes to zero for $b / a \approx 0.9$. This is due to an interference effect: The two and four particle exchange terms in 4 give contributions to the transition amplitude with the same signs as the "direct" term whereas the one and three particle exchange terms give contributions with the opposite sign; for this special value of $b / a$ the two contributions cancel each other. So we see that the PAULI principle may drastically influence the values of certain matrix elements.

For small $b / a$ values the solid curve approaches the dashed curve, as it must, because as the average distance of the alpha clusters becomes very large the antisymmetrization becomes less and less important. The same is true if $b / a$ becomes very large because, as already mentioned, due to the large relative momentum of the clusters the overlapping of the alphaparticle cluster wave functions again becomes very small. The calculations with the two $\alpha$-cluster wave function discussed above have borne out our original arguments that excepting when the penetration of the clusters in ordinary or momentum space is small, the degree of cluster overlapping is large and the behaviour of the antisymmetrized cluster function can be completely different from that of the unsymmetrized function. We repeat that these arguments are general and not restricted to the above typical example. 


\section{A one-dimensional model nucleus}

We have seen that the overlapping of clusters may destroy the naive concept of two clusters bound together; it may be that the PAULI principle changes the value of matrix elements drastically. But we want to show that one important property remains which fundamentally influences all nuclear structure and reaction problems. If a cluster with a tightly bound closed shell configuration is surrounded by many other nucleons, then even if the degree of overlapping is large this cluster correlation usually remains energetically favoured over any kind of state in which the closed shell cluster is broken up. We want to justify this conclusion in some detail.

To understand this continued energetical preference of cluster correlations we consider a simpleone-dimensional model where the essential features creating the effect can be seen especially clearly. The model consists of a FERMI sea of nucleons into which we introduce a cluster of a finite number of nucleons. The motion of the uncorrelated nucleons in the FERM sea we describe by single particle wave functions, eigenfunctions to an infinite potential well of diameter $L$. The internal motion of the nucleons in the cluster we describe also by single particle eigenfunctions, to an infinite potential well, but of diameter $l \ll L$. We have not used an oscillator potential to build up the cluster because we wish to keep the calculation simple; for the same reason we assume the total momentum of the cluster relative to the Fermi sea is zero. By these assumptions a zero point oscillation of the cluster center of mass remains, but this has no essential influence on our considerations.

The normalized wave function of our total system is now described by the following expression

$$
\Psi=N \mathfrak{U}^{\prime}\left\{\Phi_{0} \Phi_{1}\right\}
$$

( $N$ being a normalization constant)

where $\Phi_{0}$ represents the nucleons in the FERM sea and $\Phi_{1}$ the nucleons in the cluster. $\Phi_{0}$ and $\Phi_{1}$ are both antisymmetrized functions. $\mathfrak{U}^{\prime}$ describes the antisymmetrization between the FERMI sea and the cluster. The normalized wave function $\Phi_{0}$ has the form

$$
\begin{aligned}
\Phi_{0}= & \frac{1}{\sqrt{A_{0} !}}\left(\frac{2}{L}\right)^{A_{0} / 2} \mathfrak{U}\left\{\prod_{n=1}^{A_{0} / 4} \sin \left(\frac{2 \pi n x_{n}}{L}\right) \varepsilon_{n}\right\}, \\
& -\frac{L}{2} \leqq x_{n} \leqq \frac{L}{2}
\end{aligned}
$$

( $\mathfrak{U}$ being the antisymmetrisation operator).
$A_{0}$ is the number of the nucleons in the Fermi sea. The $\varepsilon_{n}$ are spin-isospin functions. Therefore to every $n$ value belong four different spin-isospin states, if we assume that all space states are filled up with four nucleons.

For the normalized wave function $\Phi_{1}$ one obtains analogous to (11)

$$
\begin{aligned}
\Phi_{1}= & \frac{1}{\sqrt{A_{1} !}}\left(\frac{2}{l}\right)^{A_{1} / 2} \mathfrak{U}\left\{\prod_{m=1}^{A_{1} / 4} \sin \left(\frac{2 \pi m x_{m}}{l}\right) \varepsilon_{m}\right\} \\
& -\frac{l}{2} \leqq x_{m} \leqq \frac{l}{2}
\end{aligned}
$$

with $A_{1}$ being the number of the nucleons in the cluster.

For simplicity we have assumed that all single particle wave funcitons in $\Phi_{0}$ and $\Phi_{1}$ vanish at the origin. That means we use only single particle wave functions with odd parity. This restriction does not influence the generality of our considerations because the following discussion can be applied in exactly the same way to even parity states. In (11) and (12) we have assumed that in the ground state of the FERMI sea and of the cluster the lowest single particle levels of the potential wells allowed by the PAULI principle are filled up.

One recognizes immediately that single particle wave functions in the cluster are changed completely by the antisymmetrization operator $\mathfrak{U}^{\prime}$ in (10) if their wave number $k_{m}=2 \pi \mathrm{m} / \mathrm{l}$ is smaller or a little larger than the wave number $K_{F}=2 \pi A_{0} / 4 L$ of the FERMr limit. On the other hand those single particle wave functions of the cluster with $k_{m}$ appreciably greater than $K_{\mathrm{F}}$ are changed only very little.

To see this in more detail we expand the single particle wave functions of the cluster in single particle wave functions of the Fermi sea. Defining $K_{n}=2 \pi n / L$ we have

$$
\begin{aligned}
\varphi\left(x ; k_{m}\right) \varepsilon_{m} & \equiv \sqrt{\frac{2}{l}} \sin \left(k_{m} x\right) \varepsilon_{m} \\
& =\sum_{n=1}^{\infty} a\left(K_{n} ; k_{m}\right) \sqrt{\frac{2}{L}} \sin \left(K_{n} x\right) \varepsilon_{m}
\end{aligned}
$$

where after some transformations we find

$$
\begin{aligned}
& \begin{aligned}
a\left(K_{n} ; k_{m}\right) & \equiv \int_{-l / 2}^{l / 2} \mathrm{~d} x \sqrt{\frac{2}{l}} \sin \left(k_{m} x\right) \sqrt{\frac{2}{L}} \sin \left(K_{n} x\right) \\
& =\frac{4}{\sqrt{l L}}(-1)^{m} \frac{k_{m}}{K_{n}^{2}-k_{m}^{2}} \sin \frac{K_{n} l}{2}
\end{aligned} \\
& \text { which is } \sqrt{\frac{l}{L}} \text { for } k_{m}=K_{n} .
\end{aligned}
$$


Now it seems convenient to write the function of eq. (10) in another form

$$
\Psi=\bar{N} \mathfrak{U}^{\prime}\left\{\Phi_{0} \Phi_{1}\right\}
$$

where the function $\bar{\Phi}_{1}$ is defined, in analogy to (12), by

$$
\bar{\Phi}_{1}=\tilde{N} \mathfrak{N} \prod_{m=1}^{A_{1} / 4} \bar{\varphi}\left(x_{m} ; k_{m}\right) \varepsilon_{m}
$$

with

$$
\begin{gathered}
\bar{\varphi}\left(x ; k_{m}\right)=\sum_{K_{n} \sum K_{\mathrm{F}}} \bar{a}\left(K_{n} ; k_{m}\right) \sqrt{\frac{2}{L}} \sin \left(K_{n} x\right), \\
\bar{a}\left(K_{n} ; k_{m}\right)=\hat{N}\left(k_{m}\right) a\left(K_{n} ; k_{m}\right) .
\end{gathered}
$$

$N\left(k_{m}\right)$ being given by the condition

$$
\sum_{K_{n} \geq K_{\mathrm{F}}}\left|\bar{a}\left(K_{n} ; k_{m}\right)\right|^{2}=1 .
$$

The functions $\bar{\varphi}\left(x ; k_{m}\right)$ are defined in a way that they are orthogonal to the functions of the filled levels of the Fermi sea; they are normalized, but they are not orthogonal to each other. So it is necessary to include a normalization constant $\tilde{N}$ in the definition of the normalized function of eq. (16).

Now we consider the two limit cases $k_{m} \gg K_{\mathrm{F}}$ and $k_{m}<K_{\mathrm{F}}$ for the discussion of the first case we will assume that the wave number difference $\Delta k$ $=2 \pi / l$ of two successive levels in the cluster is much smaller than $k_{m}-K_{\mathrm{F}}$, that is, many levels of the cluster should lie above the Fermi sea. In the first case we have

$$
\bar{a}\left(K_{n} ; k_{m}\right) \approx a\left(K_{n} ; k_{m}\right)
$$

and

$$
\bar{\varphi}\left(x ; k_{m}\right) \approx \varphi\left(x ; k_{m}\right) .
$$

So $\bar{a}\left(K_{n} ; k_{m}\right)$ has its maximum for $K_{n}=k_{m}$, where we have $\bar{a}\left(K_{n} ; k_{m}\right) \approx \sqrt{l / L}$. The wave number width of the considered level is of order $\Delta k$ because already for

$$
K_{n} \approx k_{m} \pm \pi / l
$$

$\left|\bar{a}\left(K_{n} ; k_{m}\right)\right|^{2}$ decreases by a factor 0.4 , which means that such a cluster level is really a level not influenced by the presence of the FErmi sea. Furthermore, we have for two such levels $m$ and $m^{\prime}$

$$
\left|\int_{-L / 2}^{L / 2} \mathrm{~d} x \bar{\varphi}^{*}\left(x ; k_{m}\right) \bar{\varphi}\left(x ; k_{m^{\prime}}\right)\right| \ll 1 .
$$

In the second case the situation is different. Here the presence of the FERMI sea will alter the wave functions $\bar{\varphi}\left(x ; k_{m}\right)$ drastically from the $\varphi\left(x ; k_{m}\right)$; we have

$$
\begin{aligned}
\bar{\varphi}\left(x ; k_{m}\right) & =\sum_{K_{n} \geq K_{\mathrm{F}}} \bar{a}\left(K_{n} ; k_{m}\right) \sqrt{\frac{2}{L}} \sin \left(K_{n} x\right) \\
& \approx \hat{N}\left(k_{m}\right) \int_{K_{\mathrm{F}}}^{\infty} \mathrm{d} K a\left(K ; k_{m}\right) \sqrt{\frac{2}{L}} \sin (K x)
\end{aligned}
$$

$\sim(-1)^{m}\left(-\frac{\cos \left(\left(\frac{1}{2} l+x\right) K_{\mathrm{F}}\right)}{\frac{1}{2} l+x}+\frac{\cos \left(\left(\frac{1}{2} l-x\right) K_{\mathrm{F}}\right)}{\frac{1}{2} l-x}\right)$

where to perform the approximate integration in (22) we used $K_{\mathrm{F}}-k_{m}$ much larger than $\Delta k$. It can be shown easily that (22) does not diverge for $x \rightarrow \pm l / 2$.

One sees from (22) that due to the presence of the Fermi sea so long as we consider energy levels of the cluster which are bedded deeply into the FERMI sea the single particle wave functions $\bar{\varphi}\left(x ; k_{m}\right)$ are much more smeared out over the whole space than the original single particle wave functions (13) which vanish for $|x| \geqq l / 2$. This means that the original correlations between the cluster nucleons are reduced very much by the antisymmetrization, particularly for the low energy cluster nucleons.

Furthermore, we conclude from this consideration an extension of eq. (21), namely

$$
\left|\int_{-L / 2}^{L / 2} \mathrm{~d} x \bar{\varphi}^{*}\left(x ; k_{m}\right) \bar{\varphi}\left(x ; k_{m^{\prime}}\right)\right| \ll 1
$$

with $\quad k_{m} \gg K_{\mathrm{F}}, \quad k_{m^{\prime}}<K_{\mathrm{F}}$.

We use these results for the discussion of the energy expectation value of the total system consisting of the Fermi sea and the cluster using the wave function of eq. (15). First we look for the kinetic energy. The Fermi sea functions are eigenfunctions to the kinetic energy, whereas $\nabla^{2} \bar{\varphi}\left(x ; k_{m}\right)$ is still orthogonal to the Fermi sea functions. So we obtain

$$
\begin{aligned}
\langle\Psi|T| \Psi\rangle= & \left\langle\Phi_{0}\left|\sum_{n}^{A_{0}}\left(-\frac{\hbar^{2}}{2 M} \nabla_{n}^{2}\right)\right| \Phi_{0}\right\rangle \\
& +\left\langle\bar{\Phi}_{1}\left|\sum_{m}^{A_{1}}\left(-\frac{\hbar^{2}}{2 M} \nabla_{m}^{2}\right)\right| \Phi_{1}\right\rangle
\end{aligned}
$$

where in order to keep the notation transparent we mean by the sums

$$
\sum_{n}^{A_{0}} \equiv \sum_{n=1}^{A_{0}} \text { and } \sum_{m}^{A_{1}} \equiv \sum_{m=A_{0}+1}^{A} .
$$

The first term in (24) is the kinetic energy of the FERmi sea. It is

$$
\begin{aligned}
E_{\mathrm{KIN}}^{\mathrm{F}} & \equiv\left\langle\Phi_{0}\left|\sum_{n}^{A_{0}}\left(-\frac{\hbar^{2}}{2 M} \nabla_{n}^{2}\right)\right| \Phi_{0}\right\rangle \\
& =4 \sum_{n=1}^{A_{0} / 4} \int \mathrm{d} x_{n}\left(\frac{\hbar^{2}}{2 M} K_{n}^{2} \frac{2}{L}\right) \sin ^{2}\left(K_{n} x_{n}\right) \\
= & \sum_{K_{n}=0}^{K_{\mathrm{F}}} \frac{2 \hbar^{2}}{M} K_{n}^{2} \approx \frac{2 \hbar^{2}}{M} \frac{L}{2 \pi} \int_{0}^{K_{\mathrm{F}}} \mathrm{d} \mathbf{K} K^{2}=\frac{1}{6} \frac{\pi^{2} \hbar^{2}}{M} \frac{A_{0}^{3}}{L^{2}} .
\end{aligned}
$$


The second term in (24) is the kinetic energy of the nucleons in the cluster, where the cluster wave function $\bar{\Phi}_{1}$ is influenced by the presence of the FERMI sea in the just described manner.

We consider now the potential energy of our system. If we assume two-particle forces $V_{m m^{\prime}}$ as interaction forces between the nucleons then we have for the potential energy expectation value of our system:

$$
E_{\mathrm{POT}}=\frac{1}{2}\left\langle\Psi\left|\sum_{m=1}^{A} \sum_{m^{\prime} \neq m=1}^{A} V_{m, m^{\prime}}\right| \Psi\right\rangle .
$$

If we use for our "ansatz" (15) then the potential energy splits up into the following terms

$$
\begin{aligned}
E_{\mathrm{POT}}= & \frac{1}{2}\left(\sum_{n}^{A_{0}} \sum_{n^{\prime} \neq n=1}^{A_{0}}\left\langle\Phi_{0}\left|V_{n, n^{\prime}}\right| \Phi_{0}\right\rangle\right. \\
& +2\left\langle\mathfrak{U}^{\prime}\left\{\Phi_{0} \bar{\Phi}_{1}\right\}\left|\sum_{n}^{A_{0}} \sum_{m}^{A_{1}} V_{m, n}\right| \Phi_{0} \bar{\Phi}_{1} \mid\right\rangle \\
& \left.+\left\langle\bar{\Phi}_{1}\left|\sum_{m}^{A_{1}} \sum_{m^{\prime} \neq m}^{A_{1}} V_{m, m^{\prime}}\right| \bar{\Phi}_{1}\right\rangle\right) .
\end{aligned}
$$

For the derivation of (26) it is essential that $\Phi_{0}$ and $\bar{\Phi}_{1}$ are normalized to one and their single particle wave functions are orthogonal to each other. Therefore, any exchange term between the FERMI sea and the cluster vanishes in the first and the third term of (26).

The first term of (26) represents the potential energy of the Fermi sea and the third term the potential energy of the cluster. The second term is the interaction energy of the cluster with the FERMI sea.

For the cluster calculation we assume a simplified form for our two body forces $V_{m, m^{\prime}}$. To simulate the short range character of the nuclear forces we choose attractive $\delta$-forces of the form

$$
V_{m, m^{\prime}}=-V_{0} \delta\left(x_{i}-x_{k}\right)
$$

for the two-particle interactions as is often done in nuclear physics.

If we introduce

$$
\begin{aligned}
\varrho_{0}\left(x_{1}, x_{2}\right) & =4 \sum_{n=1}^{A_{0} / 4} \frac{2}{L} \sin \left(\frac{2 \pi n x_{1}}{L}\right) \sin \left(\frac{2 \pi n x_{2}}{L}\right), \\
\varrho_{0}\left(x_{1}\right) & =\varrho_{0}\left(x_{1}, x_{1}\right)
\end{aligned}
$$

then we obtain for the potential energy of the FERMI sea (cf. Heisenberg ${ }^{7}$ )

$$
\begin{aligned}
E_{\mathrm{POT}}^{\mathrm{F}}= & \frac{1}{2} \int \mathrm{d} x_{1} \mathrm{~d} x_{2} V_{1,2}\left\{\varrho_{0}\left(x_{1}\right) \varrho_{0}\left(x_{2}\right)\right. \\
& \left.-\frac{1}{4}\left(\varrho_{0}\left(x_{1}, x_{2}\right)\right)^{2}\right\} \approx-\frac{3}{8} V_{0} \frac{A_{0}^{2}}{L} .
\end{aligned}
$$

Inspection of (27) shows that $\varrho_{0}\left(x_{1}\right)$ is identical to the number density of nucleons in the Fermi sea. In (28) it was assumed that we have many nucleons in the FERMI sea, therefore $\varrho_{0}\left(x_{1}\right)$ becomes approximately $A_{0} / L$.

By introducing

$$
\varrho_{1}(x)=A_{1} \sum_{s_{\mathrm{m}}} \sum_{t_{\mathrm{m}}} \int \mathrm{d} x_{2} \ldots \mathrm{d} x_{A 1}|\bar{\Phi}|^{2}
$$

where $x=x_{1}$, we obtain similarly to (28) for the interaction energy between the cluster and the FERMI sea

$$
\begin{aligned}
E_{\mathrm{POT}}^{\mathrm{INT}} & =-\frac{3}{4} V_{0} \int \mathrm{d} x \varrho_{0}(x) \varrho_{1}(x) \\
& \approx-\frac{3}{4} V_{0} \frac{A_{0} A_{1}}{L} .
\end{aligned}
$$

The factor $\frac{3}{4}$ in (30) appears due to cancellations from the exchange terms in the antisymmetrized functions $\Phi_{0}$ and $\bar{\Phi}_{1}$ analogous to the factor $\frac{3}{4}$ in (28). We see that $E_{\mathrm{POT}}^{\mathrm{INT}}$ does not depend on the special form of the cluster wave function $\bar{\Phi}_{1}$ as long as the number of nucleons in the Fermi sea, $A_{0}$, is sufficiently large.

To evaluate $E_{\mathrm{POT}}^{\mathrm{C}}$ we cannot proceed as before for $E_{\mathrm{POT}}^{\mathrm{F}}$ because the single particle cluster wave funtions (17) are not all orthogonal to each other.

We introduce therefore the probability to meet a cluster nucleon ${ }^{8}$ in the volume element $\mathrm{d} x_{\mathrm{r}}$ and at the same time another cluster nucleon in the volume element $\mathrm{d} x_{\mathrm{s}}$.

This probability is given by

$$
\begin{aligned}
& \omega\left(x_{\mathrm{r}}, x_{\mathrm{s}}\right) \\
& \quad=\frac{A_{1}\left(A_{1}-1\right)}{2} \sum_{s_{1}} \cdots \sum_{t_{A 1}}\left[\int \mathrm{d} x_{3} \ldots \mathrm{d} x_{A 1}\left|\bar{\Phi}_{1}\right|^{2}\right] \mathrm{d} x_{\mathrm{r}} \mathrm{d} x_{\mathrm{s}}
\end{aligned}
$$

with

$$
x_{1}=x_{\mathrm{r}}, \quad x_{2}=x_{\mathrm{s}} .
$$

With (31) we obtain for the potential energy of the cluster

$$
\begin{aligned}
E_{\mathrm{POT}}^{\mathrm{C}} & =\frac{1}{2}\left\langle\bar{\Phi}_{1}\left|\sum_{m}^{A_{1}} \sum_{m^{\prime} \neq m}^{A_{1}} V_{m, m^{\prime}}\right| \bar{\Phi}_{1}\right\rangle \\
& =-\frac{1}{2} \int \mathrm{d} x_{\mathrm{r}} \mathrm{d} x_{\mathrm{s}} V_{\mathrm{r}, \mathrm{s}} \omega\left(x_{\mathrm{r}}, x_{\mathrm{s}}\right) \\
& =-\frac{1}{2} V_{0} \int \mathrm{d} x_{\mathrm{r}} \omega\left(x_{\mathrm{r}}, x_{\mathrm{r}}\right) .
\end{aligned}
$$

7 W. Heisenberg u. W. Macke, Theorie des Atomkerns, Vorlesungsausarbeitung des M.P.I. für Physik, Göttingen 1952.

8 This probability is not as usual a physically measurable one, for when the cluster is greatly overlapped by surrounding nucleons it is not possible to say definitely which nucleons are participating in the cluster correlation at a given moment. Nevertheless $\omega_{1}\left(x_{\mathrm{r}}, x_{\mathrm{s}}\right)$ is well defined and enters in the calculation of the potential energy. 
Eq. (32) leads to quite significant results. The potential energy of the cluster depends on the integral of the two nucleon probability function for the cluster nucleons, $\omega(x, x)$. The integral has its least value if $\omega(x, x)$ is constant over the volume of the FERMI sea. It has its greatest value if $\omega(x, x)$ is sharply peaked with a large maximum value somewhere in the space. But this means that the density of cluster nucleons, $\varrho_{1}(x)$ must also be sharply peaked with a large maximum value. Hence the stronger the cluster correlation (here determined by the cluster well width parameter, $l$ ) the greater the contribution of the cluster to the potential energy ${ }^{9}$.

Opposing this tendency of the potential energy to cluster the nucleons strongly together are the kinetic energy, the PAULI principle, and the hard core of the nuclear forces [we have not included hard core forces explicitly in the calculation; clearly they do not affect the overall nature of eq. (32). But as they are the principle source of saturation we discuss their role here]. These three effects limit, often drastically, how tightly the cluster nucleons may be packed together. As we decrease the cluster well width $l$, the kinetic energy of the cluster nucleons increases due to the uncertainty principle. The Pauli principle forbids nucleons in the same spin and isospin state to come close to one another. No nucleons may come so close that they penetrate the hard core regions. Since both the PAULI principle and the hard core forces serve to limit the available volume in which the nucleons may move, they show up here mainly as an increased contribution to the kinetic energy. The nucleons will pack together as closely as possible until the rise in kinetic energy, and possibly repulsive core potential energy, offset any further gain in potential energy from the short-range attractive forces. This important conclusion shows that the clusters remain energetically favoured even when surrounded and greatly overlapped by many other nucleons.

We consider now the break-up energy of the cluster. By break-up energy we mean the energy required to remove a nucleon from the cluster and place it in one of the unoccupied states of the FERMI

\footnotetext{
${ }^{9}$ In fact it is obvious from eq. (28) that since the potential energy of a $F_{\text {ERMI }}$ sea depends on the integral of the square of the nucleon density, then we could anticipate that its potential energy is increased by introducing cluster correlations, thereby increasing the density in some parts and decreasing it in others.
}

sea, $K>K_{\mathrm{F}}$. Unfortunately, this break-up energy is very difficult to calculate for realistic cases. From our experience with light nuclei calculations we have found that the break-up energy of a cluster is usually less but of the same order of magnitude as the break-up energy of the corresponding free particle. In order to make a calculation of the breakup energy of the cluster in the Fermi sea we will make the rather special assumption that the highest occupied levels of the cluster lie well above the level of the Fermi sea. We emphasize that this assumption is not necessary for our previous conclusion about the energetical preference of the clusters; eqs. (24) to (32) are derived without it.

With this assumption we can split up the cluster function $\Phi_{1}$ as follows :

$$
\bar{\Phi}_{1}=\overline{\bar{N}} \mathfrak{U}^{\prime}(\bar{\Phi} X) .
$$

$\Phi$ consists of the antisymmetrized product of the single particle wave functions $\bar{\varphi}\left(x ; k_{m}\right) \varepsilon_{m}$ of all cluster levels except the highest ones. $A_{2}$ is the number of these lower cluster levels. $\chi$ consists of the antisymmetrized product of the single particle wave functions of the $A_{1}-A_{2}-A_{3}$ highest cluster levels. $\bar{\Phi}$ and $\chi$ are normalized to one. $\mathfrak{U}^{\prime}$ describes the antisymmetrization between $\bar{\Phi}$ and $\chi$. To keep the notation simple we always understand that before antisymmetrization the first $A_{0}$ nucleons are in the FERmi sea, the nucleons from $A_{0}+1$ to $A_{0}+A_{2}$ constitute the group $A_{2}$ of lower energy cluster nucleons, and the nucleons $A_{0}+A_{2}+1$ to $A$ constitute the group $A_{3}$ of high energy cluster nucleons.

Due to our discussion above $\chi$ can be written in good approximation as

$$
\chi \approx \frac{1}{\sqrt{A_{3} !}}\left(\frac{2}{l}\right)^{A_{3} / 2} \mathfrak{A}\left\{\prod_{u}^{A_{3}} \sin \left(k_{m} x_{m}\right) \varepsilon_{m}\right\} .
$$

The single particle wave functions in (33) are orthogonal to each other. Further they are approximately orthogonal to the single particle wave functions of the nucleons in the FERMI sea and less rigorously to the single particle wave functions in $\bar{\Phi}^{10}$. That means, this approximation for $\chi$ is very nearly orthogonal to $\Phi_{0}$ and $\overline{\bar{\Phi}}$.

10 We repeat once more the single particle wave functions in $\bar{\Phi}$ are not all orthogonal to each other. 
With this we obtain for the kinetic energy of the cluster wave function $\bar{\Phi}_{1}$ [second term in (24)]

$$
\begin{aligned}
E_{\mathrm{KIN}}^{\mathrm{C}}= & \left\langle\bar{\Phi}_{1}\left|\sum_{m}^{A_{1}}\left(-\frac{\hbar^{2}}{2 M} \nabla_{m^{2}}^{2}\right)\right| \bar{\Phi}_{1}\right\rangle \\
= & \left\langle\overline{\bar{\Phi}}\left|\sum_{m}^{A_{2}}\left(-\frac{\hbar^{2}}{2 M} \nabla_{m^{2}}^{2}\right)\right| \bar{\Phi}\right\rangle \\
& +\left\langle\chi\left|\sum_{m}^{A_{3}}\left(-\frac{\hbar^{2}}{2 M} \nabla_{m}^{2}\right)\right| \chi\right\rangle .
\end{aligned}
$$

For the second term in (35) using (34) we can write in good approximation

$$
\left\langle\chi\left|\sum_{m}^{A_{3}}\left(-\frac{\hbar^{2}}{2 M} \nabla_{m}^{2}\right)\right| \chi\right\rangle=4 \frac{\hbar^{2}}{2 M} \sum_{m}^{A_{3} / 4} k_{m}^{2} .
$$

If we now break up a cluster by removing a nucleon from one of the highest occupied cluster single particle levels to an unoccupied level of the Fermi sea, then to a good approximation the kinetic energy change will be described by the variation of the term (36) only. But as long as one neglects rearrangement effects (see for instance ${ }^{11}$ ) this kinetic energy variation is the same as in the case where one considers the breaking up of the cluster without a surrounding FERMI sea ${ }^{12}$.

We consider now the potential energy of our system, eq. (26). The contribution of the potential energy from the first two terms $E_{\mathrm{FOT}}^{\mathrm{F}}+E_{\mathrm{POT}}^{\mathrm{TNT}}$ of $(26)$ is unaltered by break-up, because as we pointed out $E_{\mathrm{POT}}^{\mathrm{INT}}$ does not depend on the density arrangement of the cluster. This means the change in the total potential energy due to break-up comes just from the change in thecluster potential energy $E_{\text {РОT }}^{\mathrm{C}}$ itself. For more detailed discussion of the cluster potential energy we again employ our ansatz for $\bar{\Phi}_{1}$ in (33). With this we obtain analogously to (26) through (30)

$$
\begin{aligned}
E_{\mathrm{POT}}^{\mathrm{C}}= & \left\langle\bar{\Phi}\left|\sum_{m}^{A_{2}} \sum_{m^{\prime} \neq m}^{A_{2}}\left(-\frac{1}{2} V_{0} \delta\left(x_{m}-x_{m^{\prime}}\right)\right)\right| \bar{\Phi}\right\rangle \\
& -\frac{3}{4} V_{0} \int \mathrm{d} x \varrho_{2}(x) \varrho_{3}(x)-\frac{3}{8} V_{0} \int \mathrm{d} x\left|\varrho_{3}(x)\right|^{2} .
\end{aligned}
$$

The first term and the third term in (37) describe the interaction among the low energy (which we shall call "inner") cluster nucleons and among the high energy ("outer") nucleons respectively. The second term represents the interaction between the outer and inner nucleons.

11 P. Mrttelstaedt, Nucl. Phys. 17, 499 [1960).
Analogously to (32) the potential energy of the inner cluster nucleons can be written as

$$
\begin{array}{r}
E_{\mathrm{POT}}^{\mathrm{INNER}} \equiv\left\langle\Phi\left|\sum_{m}^{A_{2}} \sum_{m^{\prime} \neq m}^{A_{2}}\left(-\frac{1}{2} V_{0} \delta\left(x_{m}-x_{m^{\prime}}\right)\right)\right| \bar{\Phi}\right\rangle \\
=-\frac{1}{2} V_{0} \int \mathrm{d} x_{\mathrm{r}} \omega_{2}\left(x_{\mathrm{r}}, \mid x_{\mathrm{r}}\right)
\end{array}
$$

where $\omega_{2}\left(x_{\mathrm{r}}, x_{\mathrm{s}}\right) \mathrm{d} x_{\mathrm{r}} \mathrm{d} x_{\mathrm{s}}$ is the "probability" to meet at the same time one inner cluster nucleon in the volume element $\mathrm{d} x_{\mathrm{r}}$ and another inner cluster nucleon in the volume element $\mathrm{d} x_{\mathrm{s}}$ (see however footnote ${ }^{13}$ ). From (37) and (38) we see once more that the potential energy of the cluster will become large if the nucleons in the cluster are packed as tightly as possible because (37) and (38) will become large if $\varrho_{2}(x)=\int \mathrm{d} x_{\mathrm{s}} \omega_{2}\left(x, x_{\mathrm{s}}\right)$ and $\varrho_{3}(x)$ are large in some local region of the Fermi well.

We now consider the change of the potential energy if we break up the cluster by removing a nucleon of the highest occupied cluster levels to an unoccupied state of the Fermi sea. We see from our formulas (37) and (38) that the only terms which will change are the interaction among just the outer cluster nucleons themselves and the interaction between the outer cluster nucleons and the inner cluster nucleons. Again analogously to the corresponding kinetic energy change, the interaction energy change of the outer cluster nucleon is in good approximation the same as if no Fermi sea were present as long as we neglect rearrangement effects ${ }^{11}$. The positive energy change (decreasing binding energy) is

$$
\triangle E_{\text {OUTER }}^{\mathrm{INNER}}=\frac{3}{16} V_{0} \int \mathrm{d} x\left|\varrho_{3}(x)\right|^{2} .
$$

The energy change of the interaction energy between the inner and outer cluster nucleons will be also positive and is given by

$$
\Delta E_{\mathrm{POT}}^{\mathrm{INNER}-\text { OUTER }}=\frac{3}{16} V_{0} \int \mathrm{d} x \mid \varrho_{2}(x) \varrho_{3}(x) .
$$

This energy change is smaller than the corresponding energy change of the free cluster because, as we pointed out earlier, the nucleon density $\varrho_{2}(x)$ of the inner nucleons is spread out over the whole space more in the case where a surrounding FERMI sea is present than where no surrounding FERMI is present. If one takes into account the exclusion principle between the inner cluster nucleons themselves then $\varrho_{2}(x)$ is smeared out even more, because the new single particle wave functions of the inner cluster nucleons, eq. (17), are no longer orthogonal.

12 Except that now no restriction due to the $P_{A U L I}$ principle exists as to which state outside of the cluster one can place the cluster nucleon in. To this point we return shortly. 
This decrease of the positive energy change by going from the free cluster case to the case where the cluster is surrounded by other nucleons is partly compensated by the following effects. First the negative kinetic energy change of the cluster will be larger for the free cluster than for a surrounded cluster because in the former case no states outside of the cluster are already occupied by nucleons of the FERMI sea. Second, rearrangement energy effects, which always increase the binding energy of the system, are again due to the Pauli principle usually larger in the former case than in the latter. Therefore the energy needed to break up the cluster will be of the same order of magnitude whether the cluster is surrounded by other nucleons or not ${ }^{13}$. So we see in this calculable, though special, case the trend found in light nuclei for the value of the break-up energy remains valid.

We have now to ask if the arguments for the energetical favouring of clusters, discussed following (32), are restricted to our special example or do they hold in general. The essential point was that the attractive $\delta$-forces want to pack the nucleons as tightly as possible. While we do not have $\delta$-forces in reality, we do have average attractive forces of finite but very short range. Hence (32) clearly reflects the principle trend of the real forces. A second point is that we have not included the hardcore forces explicitly. However, suppose we start with a FERMI sea of completely uncorrelated nucleons representing nuclear matter. The average density of nuclear matter is such that there is only a small probability for nucleons to come so close together that they penetrate the hard-core region ${ }^{14,15}$.

Hence, aside from determining the equilibrium density, hard core effects are not very significant for the wave function of the Fermi sea. But we have seen in (28) that the potential energy of the Fermi sea depends on the integral of the square of the density. Then we can anticipate immediately that the potential energy is increased by introducing cluster correlations, thereby increasing the density in some parts of the FERMI sea and decreasing it in others. Eventually the density in the cluster can

${ }^{13}$ In this example we have restricted ourselves to the case where the cluster as a whole is at rest. Our considerations are certainly valid also if the cluster oscillates relative to the FERMI sea. For such an oscillation increases the average kinetic energy of every cluster nucleon and therefore en- be made sufficiently great that hard-core effects will be significant, as well as the rise in kinetic energy and Pauli principle effects. But before we have reached this point we have already shown the energetical favouring for the existence of clusters. Hence the hard-core forces do not prevent the presence of clusters; rather they serve primarily only to help limit the final strength of the cluster correlations. Hence our use of simple attractive $\delta$-forces is justified.

Now let us consider the generality of our model, a cluster in a Fermi sea. The first point is that the important result (32) is not dependent on the form of the well used to build up the cluster nor on the fact that the model is not three dimensional. Although the calculation is considerably more complicated, the same result is obtained if the cluster is constructed with an oscillator well. Nor does the Fermi sea approximation introduce any essential restriction. If we wish to narrow the width of the Fermi sea and use a large but realistic number of nucleons, the same overall behaviour would recur. In fact if the larger and smaller clusters are described in the frame of the oscillator cluster model the general results are unchanged, as explicit but lengthy calculations show. In particular the potential energy contribution of the cluster remains intimately related to the density of the nucleons in the cluster.

Finally we discuss what general inferences may be drawn regarding the most energetically favoured cluster configuration and the related cluster break-up energy. We begin with a plausible suggestion which we then immediately refine. After antisymmetrization with the Fermi sea the original cluster density is reduced considerably since the cluster nucleons (particularly the "inner" nucleons) become smeared out over the Fermi sea; it is plausible that this reduced density is maximally peaked if the original cluster before antisymmetrization with the FERMI sea was as densely packed as possible. We can draw an immediate conclusion. The original cluster before antisymmetrization with the FERMI sea corresponds to a free cluster, and it is well known that free clusters are exceptionally tightly packed when they are of closed shell configuration, i. e. clusters

larges the energy difference between them and the nucleons of the FERMr sea.

14 L. C. Gomes, J. D. Walecka, and V. F. Weisskopf, Ann. Phys. N.Y. 3, 241 [1958].

15 A. de Shalit and V. F. Weisskopf, Ann. Phys. N.Y. 5, 282 [1958]. 
having only one or few neutrons and protons outside a closed (magic number) shell. If our plausibility argument is correct, a closed shell cluster in the FERMI sea will be energetically favoured over a nonclosed shell cluster because even after antisymmetrization a closed shell cluster will be more densely packed than a non-closed shell cluster. Now let us refine the argument. If we expand an arbitrary antisymmetrized cluster function in oscillator shell model functions, the "inner" (low energy) nucleons will occupy completely filled oscillator shells. Nothing more can be done to correlate these inner nucleons further without breaking up the shells. On the other hand the outer nucleons occupy unfilled shells and so can be placed in a superposition of states which packs them tightly. Now we refer back to our calculation of the break-up energy of a cluster in the FERMr sea. Under the special assumption made there (that they lie well above the Fermi sea), the single particle levels of the outer nucleons are negligibly affected by antisymmetrization with the FERMI sea. Hence they are nearly identical to those of a free cluster. Hence the outer nucleons of the cluster in the Fermi sea will be most tightly packed if the cluster is a magic number cluster. As just pointed out, since the outer nucleons are the only ones which can be significantly packed anyway, then closed shell clusters are more energetically favoured than non-closed shell clusters. Suppose we now relax our special assumption so that the outer nucleon levels need not lie far from the surface of the Fermi sea. It still remains that the outer nucleons are the ones least affected by antisymmetrization, and therefore it remains plausible that in a closed shell cluster the nucleons are packed more closely than in a non-closed shell cluster even when surrounded by other nucleons.

A further refinement gives some idea of the breakup energy of the cluster. In a shell model expansion of the antisymmetrized cluster function it is well known that the attractive nuclear forces are most strongly between nucleons in the same shell. Then the energy required to remove the last nucleon from the cluster will be greatest if the last shell is filled. However, if the last shell is filled or almost filled, the outer nucleons provide a significant part of the energy binding the last nucleon to the cluster. Since the outer nucleons are the least smeared out by the antisymmetrization, we expect for a closed shell cluster that the breake-up energy to remove the last nucleon will not be too greatly reduced from that for the corresponding free cluster.

Hence we conclude generally that closed shell clusters usually remain energetically preferred even when highly overlapped by many surrounding nucleons, and that their break-up energy is usually less but of the same order of magnitude as their break-up energy when free.

\section{Application of the results}

We will now shortly apply our results to some specific examples. In the case of light nuclei one can explicity show quantitatively, as often discussed already, that closed shell or nearly closed shell clusters, such as triton-clusters, $\alpha$-clusters, $\mathbf{O}^{\mathbf{1 6} \text {. }}$ clusters, etc. in their ground states remain energetically favoured compared with other clusters, even if they are surrounded by other nucleons. Deuteron clusters usually are even more tightly bound when they are bedded in other nucleons than when they are free particles.

In the case of heavy clusters such explicit calculations are not yet possible, and we have to rely on the results of our general considerations.

As an example of heavy cluster behaviour, let us qualitatively examine the effects of cluster overlapping on a heavy closed shell cluster within a larger nucleus. For simplicity we consider a cluster having 50 neutrons. As far as cluster overlapping is concerned we can neglect the presence of the protons. Let us assume that our heavy nucleus has a total of 132 neutrons, so that the other cluster, having 82 neutrons, is also a closed shell cluster. These particular magic number clusters are of interest in connection with asymmetric fission. As usual, to determine what remains of a cluster correlation after antisymmetrization we will consider our example simultaneously in several representations: in the oscillator shell model, in the oscillator cluster model, and with generalized cluster functions.

In the oscillator shell model the energetically lowest state allowed by the PAULI principle for 132 neutrons has the first six oscillator shells filled and the seventh partially occupied by the last 20 neutrons. This state has 540 oscillator quanta of energy. If this oscillator shell model function is now considered in conjunction with the nuclear $\mathrm{H}_{\mathrm{AMIL}}$ Tovian, the spin-orbit part of the nuclear forces strongly favour the outside nucleons to have $l$ and $s$ 
parallel with $l$ as large as possible. Hence 14 of the 20 outside neutrons will occupy the 14 possible single particle levels of the $(1 \mathrm{i})$ subshell in which $l$ and $s$ are parallel. These 14 neutrons along with the 112 neutrons filling the first six oscillator shells comprise the nuclear closed shell configuration of 126 neutrons (Goeppert-MAYER and Jensen ${ }^{16}$ ). The remaining 6 neutrons occupy other subshells of the $7^{\text {th }}$ oscillator shell.

In the oscillator cluster model we construct the internal functions of the clusters with single particle functions of an oscillator well ${ }^{17}$. Therefore due to the PAULI principle a cluster of 50 neutrons must have 130 oscillator quanta of internal energy. If we take into account the nuclear spin-orbit forces, the ten outside neutrons in the unfilled $5^{\text {th }}$ oscillator shell will occupy the 10 levels of the $1 \mathrm{~g}$ subshell in which $l$ and $s$ are alligned, thereby completing the nuclear 50 neutrons closed shell. Similarly a cluster of 82 neutrons will have 270 oscillator quanta of internal energy, and the twelve neutrons in the unfilled $6^{\text {th }}$ oscillator shell will occupy the 12 levels with $l$ and $s$ parallel of the $(1 \mathrm{~h})$ subshell. The lowest relative oscillation of the two clusters must have at least 140 oscillator quanta of energy; for we have seen in the oscillator shell model that a wave function for 132 neutrons of less than 540 oscillator quanta vanishes under antisymmetrization. If we expand the oscillator cluster function in oscillator shell model functions, then, analogously to the Fermi sea example, under antisymmetrization any terms containing two nucleons in the same single particle level vanish.

Hence our oscillator cluster function corresponds to an antisymmetrized oscillator shell model function where the first six oscillator shells are filled and the last twenty nucleons are in some superposition of single particle levels of the $7^{\text {th }}$ oscillator shell. Due to our magic number cluster assumption we expect this superposition will be such as to pack these last twenty nucleons as tightly as possible within the restriction of the PAULI principle. In the oscillator shell model these correlations between the last 20 nucleons are all that remains after antisymmetrization of our initial unsymmetrized cluster

16 M. Goeppert-Mayer and J.H.D. Jensen, Elementary Theory of Nuclear Shell Structure, Wiley \& Sons, New York 1955.

17 We use an oscillator well again to construct the internal function, instead of a square well as in the cluster plus $F_{\text {ERMI }}$ sea example, in order to facilitate comparison with the oscillator shell model. function. However, despite the fact that the PAULI principle drastically reduces the cluster correlations present in the unsymmetrized oscillator cluster function, the remaining correlations will energetically favour such a state over any other state, in which the last 20 nucleons are less correlated.

If we now leave the oscillator assumption and use generalized cluster functions, then the energetical preference for the 50 and 82 neutrons magic number cluster representation will increase. To see this, consider an expansion of the generalized cluster function in oscillator shell model functions. In this expansion will appear terms in which the 6 lowest oscillator shells are not completely filled and some higher oscillator levels are occupied. All the nucleons in unfilled oscillator shells can now be correlated so as to increase the nucleon density within the limits of the PAULI principle and by the general for of the potential energy will increase ${ }^{18}$.

The break-up energy of the clusters is difficult to estimate. If we consider a reference frame in which the oscillator well of the 82 neutron cluster is at rest (analogous to the FERMI sea in our previous example) and expand the 50 neutron cluster function, which now has all the relative motion in addition to its internal energy, in states of the $82 \mathrm{neu}$ tron cluster oscillator well, then the most energetic neutrons of the 50 neutron cluster lie only one oscillator shell above the last occupied oscillator shell of the 82 neutron cluster. Consequently the simplification of our special break-up energy calculation for the cluster in a FERMI sea does not occur. But from our general remarks we expect the breakup energy to be somewhat smaller than that of the corresponding free cluster. It approaches the breakup energy of the free clusters more and more as we increase the relative motion of the two clusters.

If we had instead considered a 50 proton closed shell cluster within a larger nucleus, our considerations would have gone similarly. The main difference would be that in heavy nuclei the break-up energy of a 50 proton cluster is even larger than that of a 50 neutron cluster, because it is surrounded by less protons than the 50 neutron cluster by neutrons.

18 We might also note here that the nucleon density will be slightly increased near the surface of the clusters due to the spin-orbit forces, which tend to favour large nucleon orbital angular momentum values when $l$ and $s$ are parallel. 